\title{
25 Research Square \\ Establishment of narrow root fracture models using modified temperature cycling method and diagnosis using different cone-beam CT units
}

Jiahao Liang

Nanjing Stomatological Hospital https://orcid.org/0000-0002-4385-2313

\section{Seyiti Pakezhati}

Nanjing Stomatological Hospital

Dantong Cao

Nanjing Stomatological Hospital

Ya Cao

Nanjing Stomatological Hospital

Xin Xie

Danyang third people's hospital

Antian Gao

Nanjing Stomatological Hospital

Zhiyong Wang

Nanjing Stomatological Hospital

Zitong Lin ( $\square$ linzitong_710@163.com )

https://orcid.org/0000-0001-9884-804X

\section{Research article}

Keywords: Cone-beam CT, Diagnosis, Fracture line, Modified temperature cycling

Posted Date: April 17th, 2020

DOI: https://doi.org/10.21203/rs.3.rs-21595/v1

License: (1) (1) This work is licensed under a Creative Commons Attribution 4.0 International License.

Read Full License 


\section{Abstract}

Background The aim of this study was to Use modified thermal cycling method to establish narrow root fracture models and evaluate the diagnosis efficiency of them using four different cone-beam CT (CBCT) units.

Methods Fifty-six intact teeth were selected and the crowns of the teeth were embedded using general purpose acrylic resin. 50 VRF models were established by soaking these teeth in liquid nitrogen and hot water cyclically; 6 teeth were used as negative control. All the 56 teeth were scanned with the smallest voxel size of four different CBCT units (NewTom VGi, Planmeca Promax 3D Max, Kavo 3D eXam and Soredex Scanora3D). 10 teeth were randomly selected and the roots were sliced using slow-speed saw to obtain horizontal root sections. Scanning electron microscope (SEM) was used to measure the width of the fracture lines (FLs). The CBCT images were evaluated for the presence or absence of fracture lines. Sensitivity, specificity, positive predictive value (PPV), negative predictive value (NPV) and area under the receiver operating characteristic curve (AUC) were calculated for the diagnosis of FLs using the four CBCT units.

Results Fifty narrow root fracture models were successfully established, and 25 root sections with 45 FLs were acquired. The width of FLs was from $3.43 \mu \mathrm{m}$ to $143 \mu \mathrm{m} ; 32.06 \%$ were less than $25 \mu \mathrm{m}$, only $9.6 \%$ were from 75 to $150 \mu \mathrm{m}$. The AUC was 0.59, 0.520, 0.61 and 0.58 for NewTom VGi, Planmeca Promax 3D Max, Kavo 3D eXam and Soredex Scanora3D, respectively.

Conclusions The modified temperature cycling method is a simple and effective method to establish narrow root fracture models, the diagnosis efficiently for these narrow fracture lines were quite poor using all the four different CBCT units.

\section{Background}

Root fracture is defined as a fracture of a tooth that involves the dentin, cementum, and pulp and they may occur in any direction or orientation [1]. X-ray films enabled clinicians to visualize the roots which are surrounded by alveolar bone, therefore provide important information for the diagnosis of root fractures and treatment planning. However, the conventional 2-dimensional X-ray films often could not give a definite diagnosis in clinical practice due to its disadvantages such as magnification, distortion and anatomic superimposition. The recently widely used CBCT, due to its three-dimensional presentation of teeth with high spatial resolution, enhanced the accuracy of diagnosis of root fractures significantly and being paid much attention to [2-6]. Although CBCT offers clear advantages over conventional X-ray films for the diagnosis of root fractures, the use of $\mathrm{CBCT}$ for the detection of root fractures remains controversial [7-9]. The complexity of fracture lines and the difference of CBCT units used in clinic are two important factors [10-12]. It's known that the ease of diagnosis will vary according to the extent of the fracture, the detection of incomplete fractures and hairline-like fractures without obvious separation of the fractured fragments could be more difficult than obvious displaced root fractures. And different 
CBCT units have different voxel size, radiation dose and reconstruction algorithm, these all may influence the quality of CBCT images and the diagnosis. To explore the influence of these two factors and establish a proper protocol of usage $\mathrm{CBCT}$ in clinic, many in vitro researches were performed. And the establishment of an artificial root fracture model with similar distribution with nature ones is the foundation of these researches. Mechanical methods were used to establish artificial VRF models in most previous studies $[8,12,13]$, the width of the fracture lines in those studies were uniform and much wider than the nature ones, especially wider than the ones at the initial stage.

The aim of this study was mainly 2-fold: firstly we modified the thermal cycling method to establish narrow root fracture models which are more similar with the nature ones at the initial stage in clinic; secondly, compared the diagnosis efficiency of four different CBCT units with the smallest voxel size for these narrow root fracture lines.

\section{Methods}

\section{Objectives collection}

Fifty-six intact teeth were extracted with minimally invasive extraction due to orthodontic treatment plan or periodontal diseases at the Nanjing Stomatological Hospital, Medical School of Nanjing University. The inclusion criteria were no visual evidence of external cracks, craze lines, or fracture with the naked eye following extraction. The exclusion criteria were the presence of dental caries, root absorption, severe abrasion, wedge-shaped defects, and horizontal or vertical root fractures. This study was approved by the Ethics Committee of Nanjing Stomatological Hospital, Medical School of Nanjing University.

\section{Establishment of artificial vertical root fracture model}

All 56 freshly extracted teeth were immediately immersed in saline solution $(0.9 \%$ isotonic $\mathrm{NaCl})$. Before establishing in vitro artificial root fracture models, the crowns of the teeth were embedded with general purpose acrylic resin (Unifast Trad, GC Corporation, Tokyo, Japan). Thereafter, 50 teeth were soaked in $100^{\circ} \mathrm{C}$ hot water for $1 \mathrm{~min}$ and then were quickly transferred into liquid nitrogen $\left(-196^{\circ} \mathrm{C}\right)$ for $1 \mathrm{~min}$, this procedure was repeated once there was one or more FLs was observed on the surface of the root of the teeth with naked eyes. All the 50 teeth were finally confirmed were positive root fracture models with the dental operating microscope (Carl Zeiss Meditec AG, S100/OPMI pico, Jena, Germany).

\section{CBCT image acquisition and evaluation}

The 56 teeth were numbered and located on a piece of polystyrene foam and then scanned using the smallest voxel size of four different CBCT units (NewTom VGi, Planmeca Promax 3D Max, Kavo 3D eXam and Soredex Scanora-3D). The details of the four CBCT units were showed in Table 1.

The CBCT images were viewed on a 29.7-in. screen MX300W LCD monitor (EizoNanao Corporation, Japan) and analyzed with inbuilt CBCT image analysis software of each unit (Planmeca Romexis Viewer 
for Planmeca Promax 3D Max, NNT Viewer for NewTom VGi, eXam Vision for Kavo 3D eXam and OnDemand3D APP for Soredex Scanora3D).

Two observers (a radiological graduate student, an experienced radiologist) evaluated all images independently. If there was a hypodense line on axial images of teeth in at least 2 consecutive slices, the tooth was diagnosed as positive one. If a consensus could not be reached between the two examiners, a senior radiologist assisted in making the final decision. Before evaluation, calibration, including unified training on diagnostic standards of fracture line was performed. After one week, the observers assessed all the images again to analyze the intra-examiner agreement.

\section{Sample preparation}

\section{Acquirement of root sections with FLs}

Ten artificial root fracture teeth were randomly selected and stabilized in square mold filled with general purpose acrylic resin (Unifast Trad, GC Corporation, Tokyo, Japan) to embed the root of the teeth. Horizontal root sections with thickness of $2 \mathrm{~mm}$ were acquired from enamel-cemental junction to the apex of root using a slow-speed saw (Isomet, Buehler, Lake Bluff, IL, USA) under water cooling. Samples without visible VRFs as well as complete fragmented were excluded. The process is shown in Fig.1. This procedure resulted in 25 root sections. All the 25 sections were numbered and marked so facial, lingual, coronal, and apical sides could be identified. During all subsequent procedures, the sections were either stored in water or kept moist to prevent preparation shrinkage fracture or crack from dehydration.

\section{Measurement of width of FLs using SEM}

The digitized images of the surface of each root section were captured with a magnification of 50x, 500x or 1000x using a scanning electron microscope (SEM, S-3400N-IIXHitachi囚Tokyo囚Japan). Widths of FLs were measured at three points (S1, S2, and S3), S1 was near to root canal, S3 was in the outer cementum surface, and S2 was the middle point of S1 and S3 (Fig. 2). These processes were operated by one operator in order to ensure the consistence of the measurements.

\section{Statistical analysis}

Statistical analysis was conducted using the SPSS 22.0 software (IBM SPSS Statistics Base Integrated Edition 22, Armonk, NY, USA). The distribution of the FLs was analyzed. Sensitivity, specificity, positive predictive value (PPV), and negative predictive value (NPV) were assessed. To determine the diagnostic abilities of the four CBCT units, receiver operating characteristic (ROC) curves was constructed and the 
areas under the ROC curve (AUC) were analyzed. Kappa analysis was used to assess inter- and intraexaminer agreement.

\section{Results}

Twenty-five root sections with $45 \mathrm{FLs}$ were acquired, and 135 points were selected and measured. The distribution of width of FLs was showed in Table 2. The widths of FLs were from $3.43 \mu \mathrm{m}$ to $143 \mu \mathrm{m}$. $14.1 \%$ points had width under $10 \mu \mathrm{m}$, while $28.1 \%$ points had width between $10-25 \mu \mathrm{m}, 9.6 \%$ were from 75 to $150 \mu \mathrm{m}$. 10 of $45 \mathrm{FLs}$ were S1 wider than S3, while the others were the opposite. Sensitivity, specificity, PPV, NPV and AUC of the four CBCT units were showed in Table 3. There was low inter- and intraexaminer agreement for the diagnosis and the results were showed in Table 4.

\section{Discussion}

The diagnosis of root fracture can be extremely challenging in clinical practice. Because of the concealed location and atypical early symptoms, its exact diagnosis or prediction commonly requires abundant of clinical experience and fine radiographic presentation. СBCT has been widely used in recent years for the detection of root fractures with relatively high accuracy and sensitivity benefit from its 3-dimensional image compared with 2-dimensional X-ray films [2,5,14]. However, the diagnosis of root fractures using CBCT was also controversial due to so many influenced factors existing (different width and direction of fracture line, variables of $\mathrm{CBCT}$ units) $[7,15,16]$. To explore a more reliable and proper standard for using CBCT to diagnosis root fractures, many in vitro studies were carried out $[3,10,13]$. And to establish an artificial root fracture model similar with the natural ones is the foundation of these studies.

There were two confusing concepts: crack and fracture. According to Rivera's review, the term 'crack' implies an incomplete break in a substance. The term 'fracture' implies a complete or incomplete break in a substance [17]. This means the crack lines could be narrower than fracture lines. And the fracture lines in the initial stage could be much narrower than those in the later stage. Therefore, the diagnosis of cracks or initial fractures could be more difficult than the diagnosis of later fractures.

There were mainly two categories of methods to produce crack or fracture lines in teeth: mechanical method and temperature control method. Mechanical methods were more commonly used to establish root fractures model. In Brady et al' study, incomplete and complete vertical root fractures were induced by inserting a sewing needle into the prepared canal and applying forces on the needle [8]. Makeeva et al. inserted posts into the root canal and gently tapped with a hammer to induce vertical root fractures [13]. Guo et al. produced vertical root fractures model by cutting the teeth using a diamond wire saw machine [12]. Lloyd et al. was firstly used the thermal cycling method to produce cracks in teeth by using a thermal cycling apparatus allowing teeth cycled between hot and cold streams of water[18]. Wang et al. created cracks by exposure teeth into liquid nitrogen after hot water at $100^{\circ} \mathrm{C}$ [19]. In this study, artificial root fracture models were established by modified thermal cycling method. The teeth were soaked in liquid 
nitrogen and hot water cyclically after their crowns were embedded with acrylic resin, this method avoids the teeth splitting completely and produced quite narrow fracture lines on the surface of the root.

The width of fracture lines produced by mechanical methods in previous study seems to be wider. In Makeeva et al. study, the width of the fracture lines ranged from 20 to 300 , the mean width of fractures in the group $50-150 \mu \mathrm{m}$ was $96.3 \pm 40.6 \mu \mathrm{m}$, while for the group $>150 \mu \mathrm{m}$ the value was $230 \pm 45 \mu \mathrm{m}$, respectively[13]. In Guo's study, the width was $189-376 \mu \mathrm{m}$ for the wide group and 110-170 $\mu \mathrm{m}$ for the narrow group, the mean width $279.5 \pm 53.6 \mu \mathrm{m}$ and $140 \pm 26.8 \mu \mathrm{m}$, respectively [12]. However, in clinical practice, the width of fracture lines, especially for initial ones, is narrower than fracture lines induced by mechanical method. Huang et al. collected 37 extracted root fracture teeth and the width of fracture lines was between 10-1070 $\mu \mathrm{m}(21.62 \%$ points was under $50 \mu \mathrm{m})$ [20]. Our study established fracture lines with width between $3.43 \mu \mathrm{m}$ to $143 \mu \mathrm{m}$; and $14.1 \%$ points was under $10 \mu \mathrm{m}, 28.1 \%$ points was between 10-25 $\mu \mathrm{m}$. This was much narrower than the fracture lines induced by mechanical methods and could simulate the initial stage of fractures.

In the current study, although the smallest voxel size was used, the diagnosis efficiently for these narrow FLs was still quite poor using all the four different CBCT units. In our study, the highest sensitivity was 0.38 which was obtained with Planmeca Promax 3D Max, and the highest specificity was 0.75 which was obtained with Kavo 3D eXam. The biggest AUC was only 0.61 with was obtained with Kavo 3D eXam, which was much lower than the previous studies $[5,15,21]$. Considering that our models were nonendonotically treated, there were no artefacts resulting from endodontic filling. The width of most fracture lines was much lower than the voxel size of CBCT should be the main reason of the low diagnosis efficiency in this study. For narrow root fractures, the current СВCT units still have great limitation in the diagnosis (Fig. 3). Therefore, it is possible that some root fractures may not be diagnosed at the time of initial because the fractures are quite narrow.

Moreover, the fracture lines in our study were not consistent and uniform which was showed by SEM. This inconsistence at different points was similar with the condition in clinic [20], fracture lines of 37 extracted VRF teeth showed inconsistent fracture space in Huang's study. This phenomenon indicated that the relationship between the width of FLs and voxel size of CBCT is more complicated than we assumed. If the FLs are much wider than the voxel size of CBCT, the detection of FLs could be of course easy, but if the width of FLs is close to or narrower than the voxel size, the condition could be sophisticated. The FL might be detected due to the wide part of it and the FL may be presented as blurred and ambiguous on CBCT images.

\section{Conclusions}

In this study, we modified thermal cycling method to establish artificial root fracture models; the widths of FLs were quite narrow and could stimulate the initial condition of fracture lines. The overall diagnosis efficiently for these narrow fracture lines was quite poor using current CBCT units. The relationship 
between the width of FLs and voxel size of CBCT is more complicated due to the complexity of fracture lines.

\section{Abbreviations}

CBCT: Cone-beam CT; SEM: Scanning electron microscope; FLs: Fracture lines; PPV: Positive prediction value; NPV: Negative prediction value; ROC: Receiver operating characteristic; AUC: Area under the receiver operating characteristic curve

\section{Declarations}

\section{Ethics approval and consent to participate}

This study was approved by the Ethics Committee of Nanjing Stomatological Hospital, Medical School of Nanjing University (2015NL-044(KS). Informed consent was obtained from all participants.

\section{Consent for publication}

Not applicable.

\section{Availability of data and materials}

The datasets used and/or analyzed during the current study are available from the corresponding author upon reasonable request.

\section{Competing interests}

The authors declare that they have no competing interests.

\section{Funding}

This work was supported by the Second Level Fund for the Young Talents in the Health Field of Nanjing City (QRX17079).

\section{Authors' contributions}


All the authors made substantial contributions to the present study. JHL \& SP wrote the manuscript;DTC, YC \& JHL carried out the experiment; XX, ATG \& SP analyzed the data and interpreted the results; ZYW collected and selected the extracted teeth; ZTL contributed the concept and design of this study. All authors read and approved the final manuscript.

\section{Acknowledgments}

Not applicable.

\section{References}

1. Abbott PV. Diagnosis and management of transverse root fractures. Dent Traumatol. 2019;35:33347.

2. Wang P, Yan XB, Lui DG, Zhang WL, Zhang Y, Ma XC. Detection of dental root fractures by using cone-beam computed tomography. Dentomaxillofac Radiol. 2011;40:290-8.

3. da Silveira PF, Vizzotto MB, Liedke GS, da Silveira HL, Montagner F, da Silveira HE. Detection of vertical root fractures by conventional radiographic examination and cone beam computed tomography - an in vitro analysis. Dent Traumatol. 2013;29:41-6.

4. Kajan ZD, Taromsari M. Value of cone beam ct in detection of dental root fractures. Dentomaxillofac Radiol. 2012;41:3-10.

5. Long H, Zhou Y, Ye N, Liao L, Jian F, Wang Y, Lai W. Diagnostic accuracy of cbct for tooth fractures: A meta-analysis. J Dent. 2014;42:240-8.

6. Cohenca N, Silberman A. Contemporary imaging for the diagnosis and treatment of traumatic dental injuries: A review. Dent Traumatol. 2017;33:321-8.

7. Chavda R, Mannocci F, Andiappan M, Patel S. Comparing the in vivo diagnostic accuracy of digital periapical radiography with cone-beam computed tomography for the detection of vertical root fracture. J Endod. 2014;40:1524-9.

8. Brady E, Mannocci F, Brown J, Wilson R, Patel S. A comparison of cone beam computed tomography and periapical radiography for the detection of vertical root fractures in nonendodontically treated teeth. Int Endod J. 2014;47:735-46.

9. Patel S, Brown J, Semper M, Abella F, Mannocci F. European society of endodontology position statement: Use of cone beam computed tomography in endodontics: European society of endodontology (ese) developed by. Int Endod J 2019.

10. Ozer SY. Detection of vertical root fractures by using cone beam computed tomography with variable voxel sizes in an in vitro model. J Endod. 2011;37:75-9. 
11. Ma RH, Ge ZP, Li G. Detection accuracy of root fractures in cone-beam computed tomography images: A systematic review and meta-analysis. Int Endod J. 2016;49:646-54.

12. Guo XL, Li G, Zheng JQ, Ma RH, Liu FC, Yuan FS, Lyu PJ, Guo YJ, Yin S. Accuracy of detecting vertical root fractures in non-root filled teeth using cone beam computed tomography: Effect of voxel size and fracture width. Int Endod J. 2019;52:887-98.

13. Makeeva IM, Byakova SF, Novozhilova NE, Adzhieva EK, Golubeva GI, Grachev VI, Kasatkina IV. Detection of artificially induced vertical root fractures of different widths by cone beam computed tomography in vitro and in vivo. Int Endod J. 2016;49:980-9.

14. Edlund M, Nair MK, Nair UP. Detection of vertical root fractures by using cone-beam computed tomography: A clinical study. J Endod. 2011;37:768-72.

15. Talwar S, Utneja S, Nawal RR, Kaushik A, Srivastava D, Oberoy SS. Role of cone-beam computed tomography in diagnosis of vertical root fractures: A systematic review and meta-analysis. J Endod. 2016;42:12-24.

16. Corbella S, Del Fabbro M, Tamse A, Rosen E, Tsesis I, Taschieri S. Cone beam computed tomography for the diagnosis of vertical root fractures: A systematic review of the literature and meta-analysis. Oral Surg Oral Med Oral Pathol Oral Radiol. 2014;118:593-602.

17. Rivera EM, Walton RE. Longitudinal tooth fractures: Findings that contribute to complex endodontic diagnoses. Endodontic Topics. 2009;16:82-111.

18. Lloyd BA, McGinley MB, Brown WS. Thermal stress in teeth. J Dent Res. 1978;57:571-82.

19. Wang S, Xu Y, Shen Z, Wang L, Qiao F, Zhang X, Li M, Wu L. The extent of the crack on artificial simulation models with cbct and periapical radiography. PLoS One. 2017;12:e0169150.

20. Huang CC, Chang YC, Chuang MC, Lin HJ, Tsai YL, Chang SH, Chen JC, Jeng JH. Analysis of the width of vertical root fracture in endodontically treated teeth by 2 micro-computed tomography systems. J Endod. 2014;40:698-702.

21. Fcs S, Kobayashi-Velasco S, Braga MM, Mgp C. Radiographic diagnosis of root fractures: A systematic review, meta-analyses and sources of heterogeneity. Dento Maxillo Facial Radiology. 2017;46:20170400.

\section{Tables}

Table 1 Exposure parameters of four CBCT units

\begin{tabular}{cccccc}
\hline CBCT units & FOV $(\mathrm{cm})$ & Voxel size $(\mu \mathrm{m})$ & Tube voltage $(\mathrm{kVp})$ & Tube current(mA) & Scan time $(\mathrm{s})$ \\
\hline NewTom VGi & $5 * 5$ & 100 & 110 & 7.48 & 6 \\
\hline Planmeca Promax 3D Max & $5 * 5$ & 100 & 80 & 6.3 & 12.1 \\
\hline Soredex Scanora3D & $5 * 5$ & 100 & 90 & 10 & 13.6 \\
\hline Kavo 3D eXam & $8 * 8$ & 125 & 120 & 5.0 & 5 \\
\hline
\end{tabular}


Table 2 Distribution of width of FLs using SEM

\begin{tabular}{lcccccc}
\hline & \multicolumn{6}{c}{ Width of fracture lines $(\mu \mathrm{m} \square$} \\
\cline { 2 - 7 } & $<10$ & $10-25$ & $25-50$ & $50-75$ & $75-125$ & $125-150$ \\
\hline No. of points & 19 & 38 & 45 & 20 & 12 & 1 \\
\hline Frequency (\%) & 14.1 & 28.1 & 33.3 & 14.9 & 8.9 & 0.7 \\
\hline
\end{tabular}

Table 3 Sensitivity, specificity, PPV, NPV and AUC of the four CBCT units

\begin{tabular}{lllll}
\hline & NewTom & Planmeca & Soredex & Kavo \\
\hline Sensitivity & 0.24 & 0.38 & 0.29 & 0.19 \\
Specificity & 0.45 & 0.16 & 0.55 & 0.75 \\
PPV & 0.65 & 0.47 & 0.72 & 0.82 \\
NPV & 0.13 & 0.12 & 0.16 & 0.13 \\
AUC & 0.60 & 0.52 & 0.59 & 0.61 \\
\hline
\end{tabular}

PPV: positive predictive value, NPV: negative predictive value, AUC: area under the ROC curve

Table 4 The reliability of the 2 examiners with regard to VRF detection

\begin{tabular}{ccc}
\hline & Intra-observer Kappa & Inter-observer Kappa \\
\hline NewTom & 0.509 & 0.283 \\
Planmeca & 0.751 & 0.106 \\
Soredex & 0.509 & 0.211 \\
Kavo & 0.559 & 0.316 \\
\hline
\end{tabular}

\section{Figures}




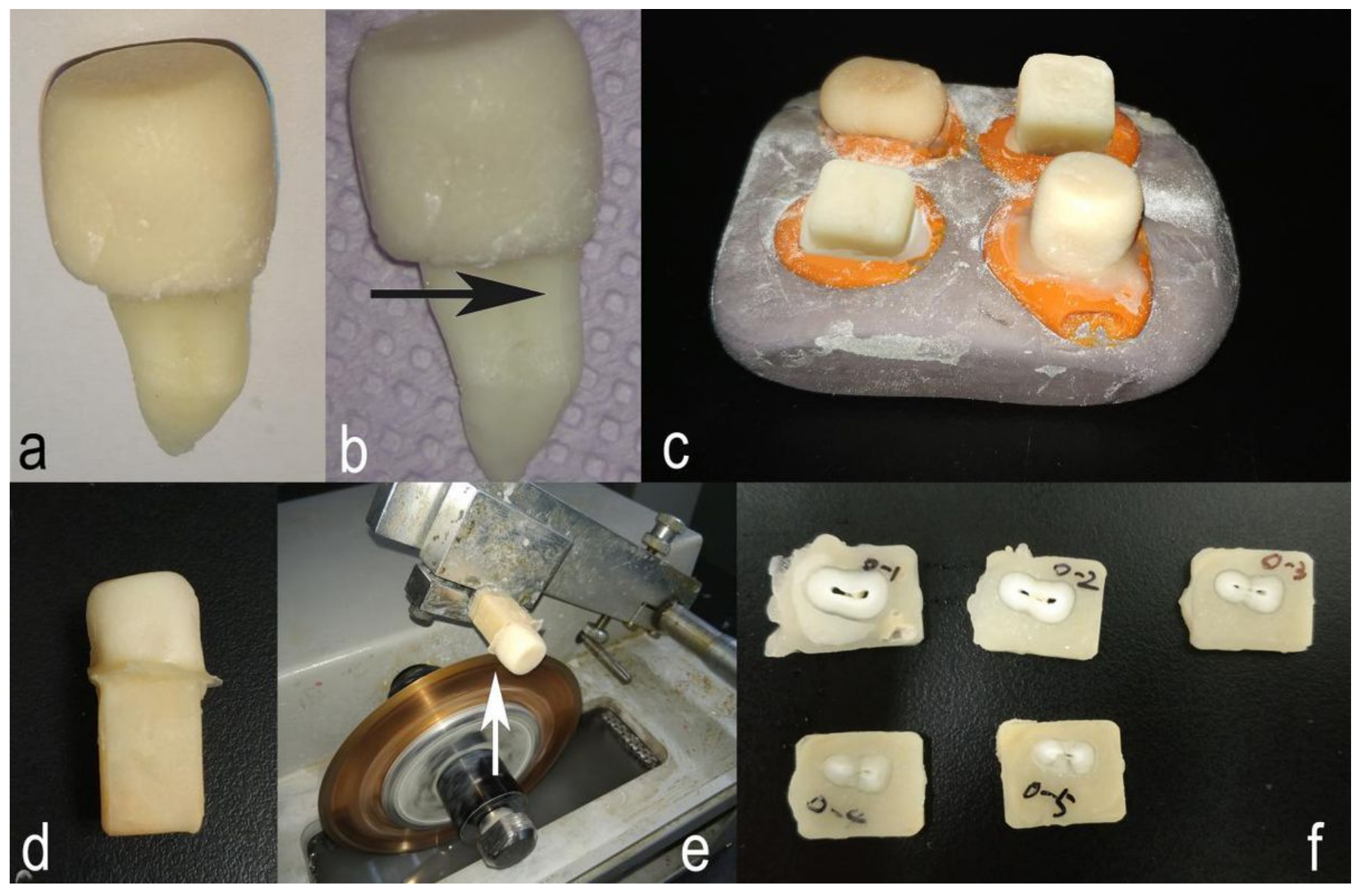

\section{Figure 1}

(a) The crown of the teeth was embedded with general purpose acrylic resin. (b)Fracture line was observed on the surface of the root after soaking these teeth in liquid nitrogen and hot water cyclically. (c)The roots of the teeth were embedded with acrylic resin in a square mold. (d)The whole tooth was embedded with acrylic resin. (e)Horizontal root sections were acquired using a slow-speed saw. (f) The root sections with fracture lines were marked and numbered. 


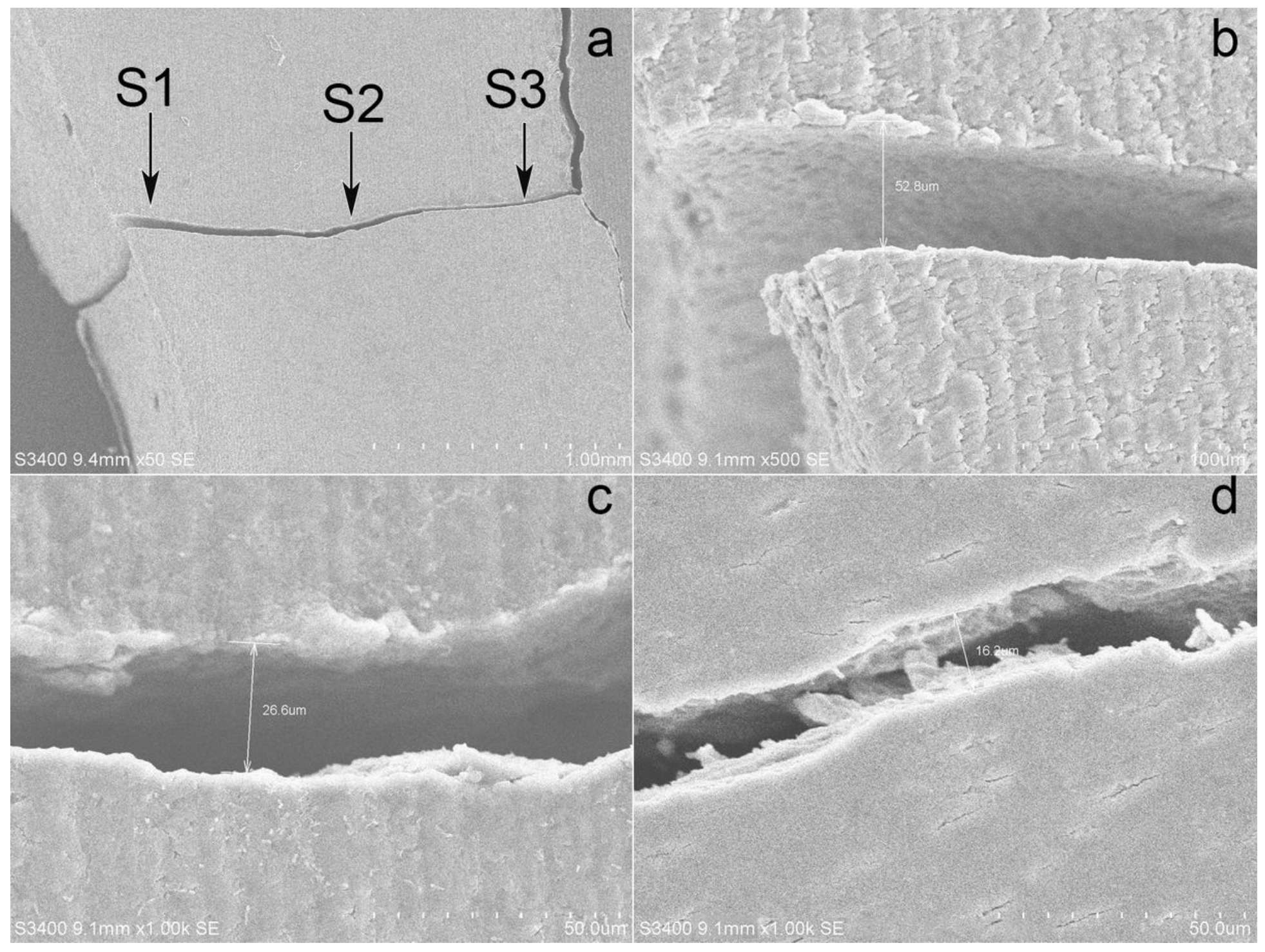

Figure 2

Widths of fracture lines measured at three points (S1, S2, and S3). 


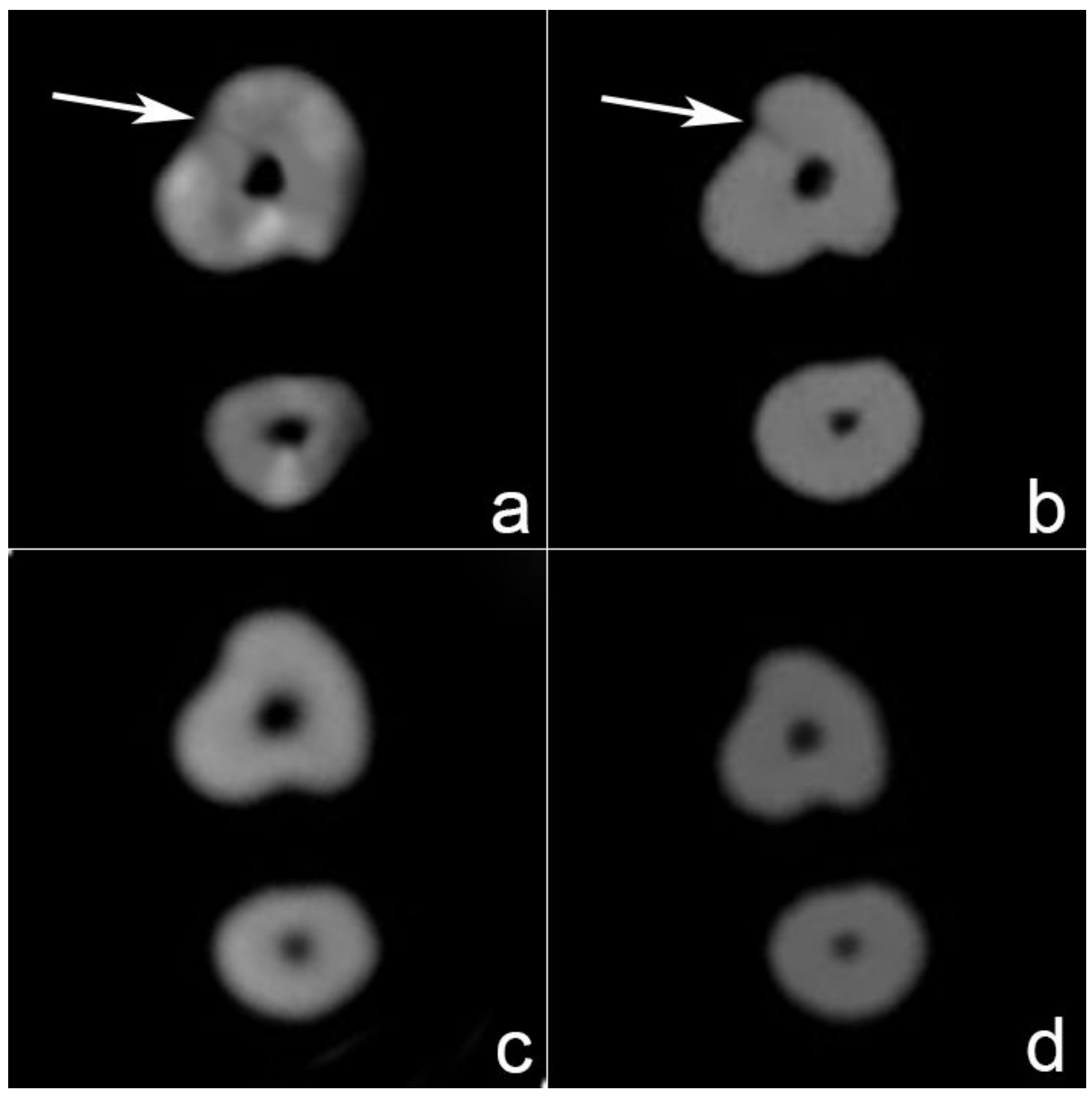

\section{Figure 3}

Example CBCT axial images of one tooth with root fracture scanned with the four CBCT units. $(a, b)$ Fracture lines could be found on images scanned with $100 \mu \mathrm{m}$ voxel size of Planmeca Promax 3D Max and $100 \mu \mathrm{m}$ voxel size of NewTom VGi. (c, d) No fracture lines could be found on images scanned with

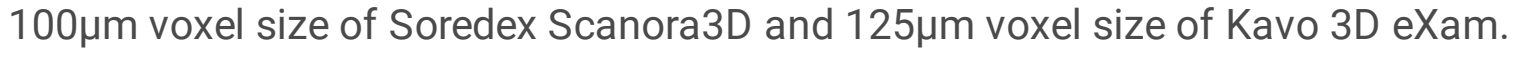

